\title{
Depletion uncertainty analysis to the MYRRHA fuel assembly model
}

\author{
Augusto Hernandez Solis ${ }^{1}$, Alexey Stankovskiy ${ }^{1, *}$, Luca $_{\text {Fiorito }}{ }^{1}$, and Gert Van den Eynde ${ }^{1}$ \\ ${ }^{1}$ SCK CEN, Boeretang 200, B-2400 Mol, Belgium
}

\begin{abstract}
In this work, the objective is to perform an uncertainty analysis on a MYRRHA -Rev.1.6 irradiation cycle study, being applied to a depletion scenario of a single fresh fuel assembly while assuming reflective boundary conditions. Such analysis is statistically based on the application of Wilk's method of building tolerance limits after 100 depletion calculations were performed with the SERPENT2 code. Due to the computational burden of such type of simulations, this propagation of nuclear data covariances study (allowed by the fast computational performance of SERPENT2) was done at constant power, constant flux and, in a final exercise, at constant power with the addition of fission yield uncertainties (all of these cases employed ENDF/B-VII.1 data). It was observed that while depleting at constant power, the statistical variation of key fission products such as ${ }^{148} \mathrm{Nd}$ is almost not present because of the normalization factor applied to the flux. In contrast, the irradiation at constant flux reveals dependence on burnup. Finally, the added fission yield uncertainties make clear the fact that they directly impact the degree of final uncertainty computed for fission products exemplified by ${ }^{148} \mathrm{Nd}$ and ${ }^{135} \mathrm{Xe}$ important for burnup estimation and reactor operation, respectively.
\end{abstract}

\section{Introduction}

MYRRHA (Multi-purpose hYbrid Research Reactor for High-tech Applications) is a flexible experimental Accelerator Driven System (ADS) in development at SCK CEN in Belgium [1]. The 1.6 revision of its core design [2] is foreseen of being able to work both in sub-critical and critical modes. On the one hand, fast spectrum material irradiation takes place in the irradiation positions (In-Pile Sections, IPS) close to the center of the core while, on the other hand, radio-isotope production by means of a thermal spectrum takes place in water-cooled IPS located at the core periphery. A series of neutronic studies simulating evolution of the cores from their initial loadings to the quasi-equilibrium conditions determined an optimum multi-zone layout with batches of fuel assemblies of different burnup level [3]. The MYRRHA core in its critical configuration at the beginning of equilibrium cycle (BoC) consists of 108 (18 batches of 6 preserving hexagonal symmetry) highly-enriched MOX fuel assemblies (FA), 6 control rods (CR) banks and 3 SCRAM rod bundles, 4 IPS and 2 outer rings of reflector/shielding sub-assemblies (as depicted in Figure 1).

Fuel cycle loading studies have been performed with the in-house depletion code ALEPH2 [4]. An "in-to-out" FA's shuffle scheme results in the burnup at discharge 52 $\mathrm{MWd} / \mathrm{kgHM}$ after 1440 days of operation, divided into a three-month irradiation cycles at a certain power, as shown in Figure 2.

ALEPH2 is wrapped around MCNP general purpose radiation transport code [5] that is used to obtain the flux spectra needed by internal ALEPH2 modules to compute

*e-mail: alexey.stankovskiy@sckcen.be

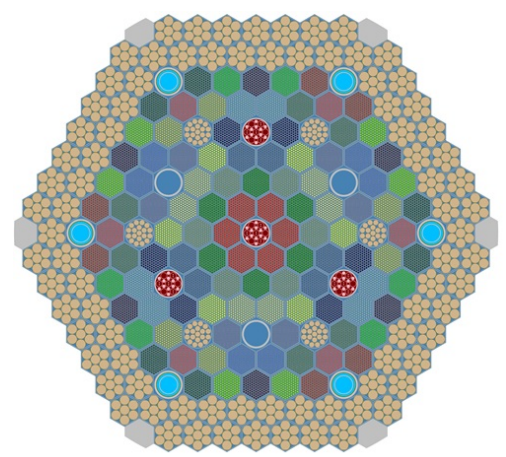

Figure 1. MYRRHA Rev.1.6 critical core layout
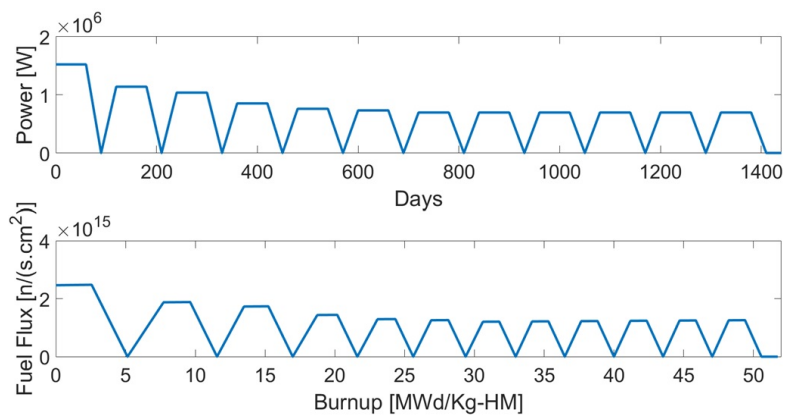

Figure 2. Irradiation analysis of the fuel loading cycle of MYRRHA Rev.1.6

reaction rates and perform the material depletion. Unfortunately, MCNP neutron transport is a bottleneck making such best estimate irradiation performance simulation 
very time consuming, and the application of a statisticallybased uncertainty analysis where the propagation of basic nuclear reaction covariances (that are encountered in the major nuclear data libraries (NDL) around the world) would result prohibitive if many code runs are necessary for applying minimum sample rules. Such is the case as, for instance, the formation of tolerance intervals (based on Wilk's formula [6]). Even for the depletion of a single fuel assembly (carrying a certain fuel batch-vector composition) while considering radial reflective boundary conditions, the use of ALEPH 2 for a sample of 100 calculations of neutronic observables of interest during a depletion analysis would be computationally very costly.

Instead, this paper describes a study performed with the SERPENT2 code [7], which includes many reactorphysics oriented modules as well as depletion capabilities. Neutron transport solver in SERPENT2 is much faster than MCNP so that a performance benchmark between SERPENT2 and ALEPH2 for a depletion calculation of a MOX fuel pin after 1640 irradiation-days at constant power resulted a factor of 5 speedup [8]. This makes feasible to perform a depletion uncertainty analysis with SERPENT2. Therefore, the main objective of this work is to propagate reaction cross-section covariances through a 1440 days depletion characteristic of a MYRRHA fresh fuel assembly with reflective boundary conditions, in order to estimate the uncertainty in time-dependent observables such as effective multiplication factor $\mathrm{k}_{\mathrm{eff}}$ as well as inventories of key nuclides. The NDL and its covariances that were employed corresponded to the ENDF/B-VII.1 library [9]. Randomized point-wise libraries (as a function of energy, both for direct and double-differential cross-sections) in the ENDF format were obtained with the help of the SANDY code [10]. Such study has been performed both at constant power and constant flux, where the behavior of nuclide uncertainty was scrutinized. Thereafter, the inclusion of uncertainties in the fission yields (to the case of depletion at constant power), added an extra value to the observed output uncertainty results based on 100 calculations with randomized NDL.

\section{MYRRHA assembly model in SERPENT2}

A radial (XY) and axial (YZ and XZ) cross-sectional plots of the MYRRHA assembly can be found below in Figure 3. The whole assembly including springs, sleeves, insulators, core restrain system and even the above core structure has been taken into account.

A comparison of the spectra of such assembly calculated both with MCNP (version 6.2 [5]) and SERPENT2 can be seen in Figure 4. Very good agreement can be observed between both codes, verifying that SERPENT2 is in fact reliable while performing neutron transport calculations. On the other hand, Table 1 shows the parameters and $\mathrm{k}_{\mathrm{eff}}$ of the SERPENT2 calculation.

\section{The SANDY code}

First, it might be convenient to briefly describe the methodology behind SANDY. This code is a numerical
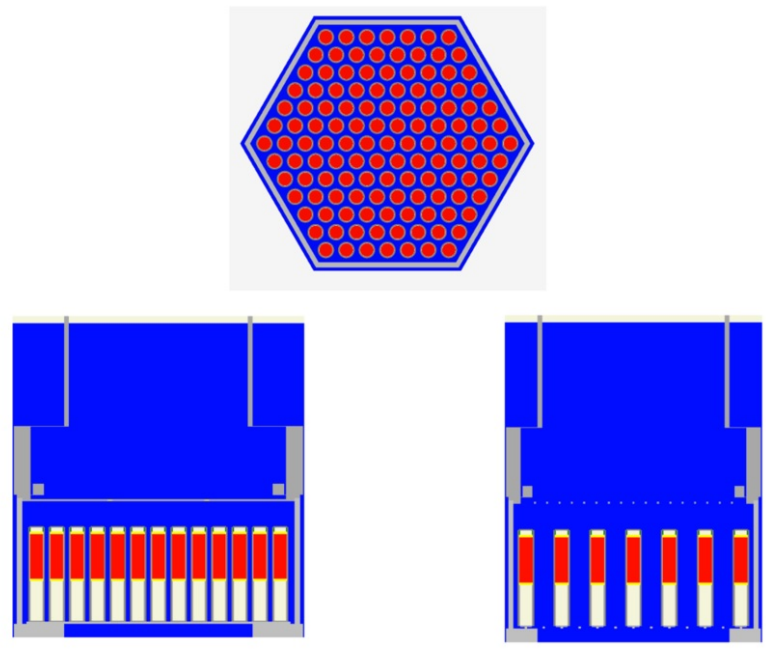

Figure 3. Cross-sectional geometry of a full MYRRHA assembly modeled with SERPENT2

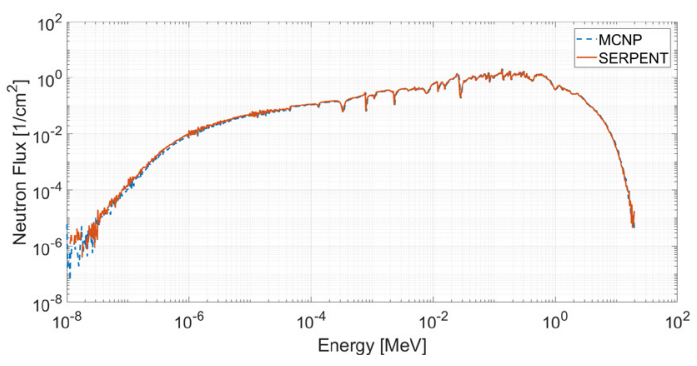

Figure 4. Normalized flux spectra comparison for a fresh MYRRHA fuel assembly

Table 1. Neutron transport calculation characteristics for SERPENT2

\begin{tabular}{ll}
\hline Boundary conditions & $\begin{array}{l}\text { Radial reflective; } \\
\text { Axial vacuum }\end{array}$ \\
Active cycles & 250 \\
Number of neutrons / cycle & 10,000 \\
$\mathrm{k}_{\mathrm{eff}}$ & $1.35585+/-0.00070$ \\
\hline
\end{tabular}

tool employed to perform the random sampling of the parameters stored in NDL's. Since it can read and process any library file in the ENDF-6 format, the tool is compatible to most of the libraries provided by the international data groups, including the general-purpose libraries ENDF/B [9], JEFF [11], and JENDL [12]. SANDY's random samples are written in perturbed copies of the original ENDF-6 file and can be used as inputs for a Monte Carlobased uncertainty propagation. The advantages of using a sampling-based tool like SANDY, rather than algorithms based on perturbation theory are clear. SANDY can work with any nuclear physics model and solver as long as the nuclear data that they compute is on the ENDF-6 format. The responses calculated with SANDY take into account first and higher order effects and are not limited by constraints of linearity. 
SANDY can sample covariance matrices from crosssections $(\mathrm{MF}=3)$, resonance parameters $(\mathrm{MF}=2)$, fission neutron multiplicities $(\mathrm{MF}=1)$, secondary particle angular distributions $(\mathrm{MF}=4)$, secondary particle energy distributions $(\mathrm{MF}=5)$, radioactive decay and fission yields $(\mathrm{MF}=8)$.

The variables that are meant to be sampled with SANDY assumed to fit a normal (or LogNormal) multivariate probability density function (PDF), where the mean value corresponds to the nominal estimate from the data, and the standard deviation is extracted from the diagonal of the covariance matrix. The simple random sampling procedure is carried out in SANDY by performing a Cholesky decomposition of the correlation matrix $\boldsymbol{C}$, in order to calculate the matrix $\boldsymbol{C}$ that fulfills the following relation:

$$
C=L L^{T}
$$

Where the correlated normally distributed samples $\boldsymbol{X}_{\boldsymbol{N}(\boldsymbol{\theta}, \boldsymbol{C})}$ are related to a matrix formed by $n_{s}$ independent sets of $n$ correlated samples from a standard normal distribution (e.g. $N(0,1))$ such as:

$$
\boldsymbol{X}_{N(0, C)}=L \boldsymbol{X}_{N(0,1)}
$$

SANDY also ensures that the extracted covariance from the original file is positive-definite and returns a lower triangle matrix $L$ that is unique with real and positive diagonal elements. More info about this methodology, and in general about SANDY can be found in [10].

\section{Results of the depletion uncertainty analysis}

The nuclides to which SANDY randomized their correspondent microscopic cross-section covariances based on ENDF/B-VII.1 can be found in Table 2. After 100 depletion calculations were performed, an independent analysis of the output observables of interest (which are now considered to be random variables) could be done by applying Wilk's theory of double sided tolerance intervals. This states that $95 \%$ of the population of a certain observable can be inferred to be covered with a $95 \%$ confidence, independently from the type of distribution such random variable is coming from [6]. Our motivation of applying such statistical theory is because 100 depletion calculations that were done for the scenario depicted in Figure 2 (and at each step a transport calculation is performed based on the parameters listed in Table 1) took almost 37 hours to be completed. Since one of the objectives of this work is to show a comparison between depleting at constant power, at constant flux and at constant power including fission yield uncertainties, longer runs to try to converge the variance uncertainty of each observables would had become very computational expensive. Therefore, by applying the use of tolerance intervals inference, some statistical deductions can already be drawn after 100 calculations along with a bearable computational time of the results.
Table 2. List of the nuclides with uncertain parameters

\begin{tabular}{lllll}
\hline $\mathrm{O}-16$ & $\mathrm{~Pb}-206$ & $\mathrm{~Pb}-207$ & $\mathrm{~Pb}-208$ & $\mathrm{Bi}-209$ \\
$\mathrm{U}-235$ & $\mathrm{U}-238$ & $\mathrm{Pu}-239$ & $\mathrm{Pu}-240$ & $\mathrm{Pu}-241$ \\
$\mathrm{Pu}-242$ & $\mathrm{Am}-241$ & $\mathrm{Am}-242 \mathrm{~m}$ & $\mathrm{Cm}-242$ & $\mathrm{Cm}-243$ \\
$\mathrm{Cm}-244$ & $\mathrm{Cm}-245$ & $\mathrm{Cm}-246$ & & \\
\hline
\end{tabular}

\subsection{Uncertainty analysis by depleting at constant power}

The first set of results were obtained by depleting at constant power for a certain period of time (and by using the default SERPENT2 settings of constant extrapolation for the predictor and linear interpolation for the corrector). A 90-day irradiation cycle was divided into 3 equal substeps; shutdown periods between cycles were 30 days. By doing this 12 times, a total of 1440 irradiation days would give a burnup of around $52 \mathrm{MWd} / \mathrm{kgHM}$. The starting power of the assembly was considered to be $1.522 \mathrm{MW}$, and it concluded at the end of irradiation with $0.6 \mathrm{MW}$, as depicted in Figure 2.

After 100 calculations, relative standard deviations (STD's) of observables of interest are shown in Figure 5 . As it can be seen for ${ }^{148} \mathrm{Nd}$, its uncertainty is negligible as a function of irradiation, and it is not following its predecessor statistical variability during burnup. The reason is that at constant power fission rate remains constant:

$$
\begin{gathered}
P=\langle\varphi\rangle \sum_{i} N_{i}\left\langle\sigma_{f}\right\rangle_{i} Q_{f, i} \\
P=\text { const } \longrightarrow\left\langle\sigma_{f}\right\rangle_{i}\langle\varphi\rangle=\text { const }
\end{gathered}
$$

Here $P$ is the total power, $N_{i}$ denotes the inventory of fissile nuclide $i,\left\langle\sigma_{f}\right\rangle_{i}$ stands for the spectrum-averaged fission cross section, $Q_{f, i}$ is the fission energy release and $\langle\varphi\rangle$ is the neutron flux. Fission product inventories (activities) $A_{F P}$ are therefore obtained as

$$
A_{F P}=Y_{F P}\langle\varphi\rangle \sum_{i} N_{i}\left\langle\sigma_{f}\right\rangle_{i}
$$

Here for simplicity we consider direct fission product yields $Y_{F P}$ only. Obviously, any perturbation in actinide's fission cross section will be compensated by the changes in neutron flux. Therefore, especially for long-lived or stable fission products, their inventory will predominantly be sensitive to fission yields.

\subsection{Comparison of uncertain observables while depleting at constant flux}

Constant flux irradiation is needed to keep the irradiation conditions in IPS unchanged during the cycle. This is basic option in case of sub-critical core operation by adjusting the proton beam current. The fuel irradiation at constant flux compared to the irradiation at constant power is depicted in Figure 6. In this case, the ${ }^{148} \mathrm{Nd}$ uncertainty becomes almost one order of magnitude higher, although still being below $1 \%$. 

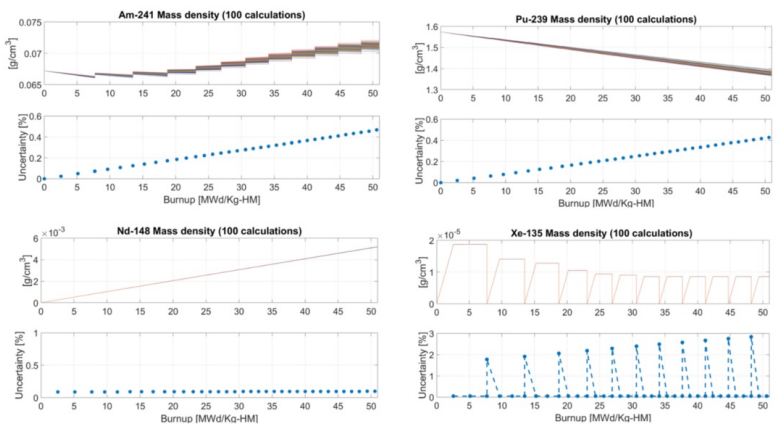

Figure 5. Nuclide inventory uncertainties (depleting at constant power)

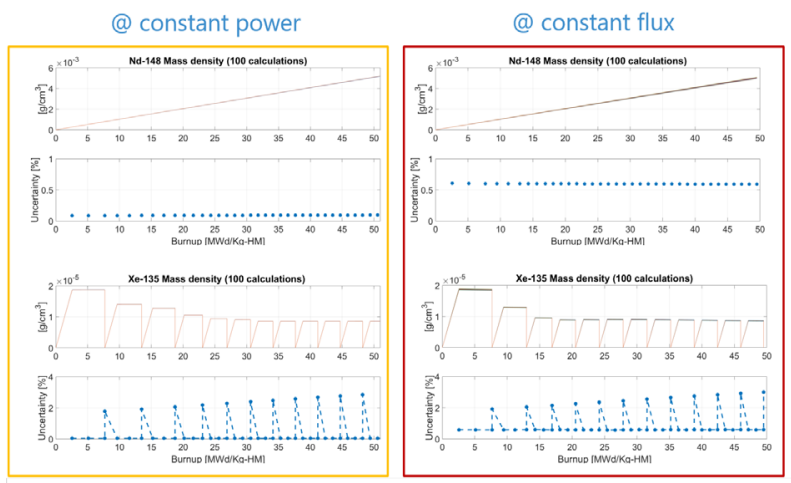

Figure 6. Comparison of fission product uncertainties obtained at constant power and constant flux

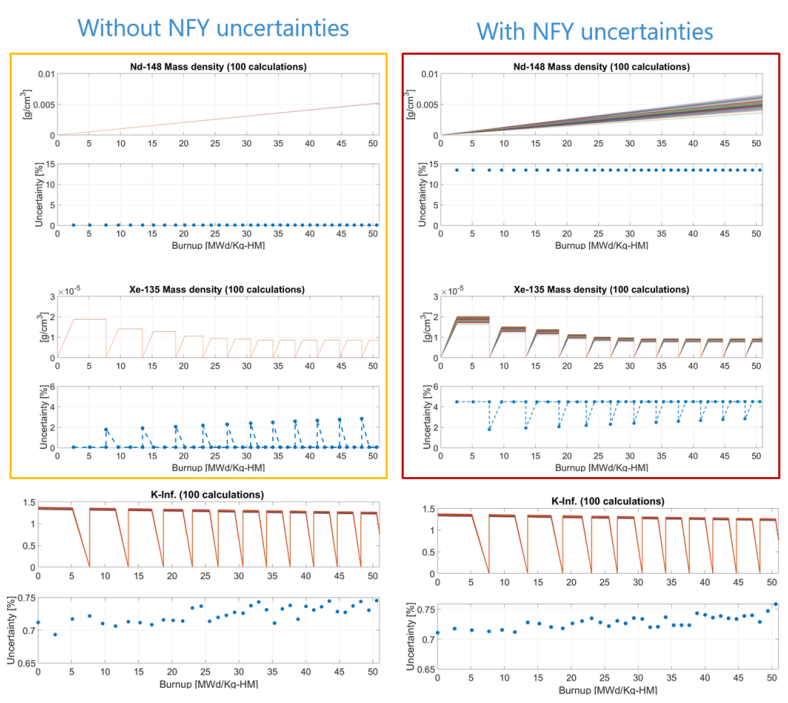

Figure 7. Uncertainties with and without account of FY uncertainties (at constant power)

\subsection{Account of fission yield uncertainty while depleting at constant power}

The corresponding fission yield (FY) uncertainties from ENDF/B-VII.1 fission yield files were included to confirm the conclusion made above in Section 4.1 on their direct effect in the case of depleting at constant power. Figure 7 compares the degree of uncertainty achieved by some nuclide observables as well as for $\mathrm{k}_{\mathrm{eff}}$. It can be seen the clear impact that adding such FY uncertainties have in the statistical variability of ${ }^{135} \mathrm{Xe}$ and ${ }^{148} \mathrm{Nd}$ but not in integral parameters such as the effective multiplication factor. Thus, by including FY uncertainties in the calculation (but not their respective correlations among them), a jump of important nuclide relative standard deviations such as burnup indicator ${ }^{148} \mathrm{Nd}$ and poison ${ }^{135} \mathrm{Xe}$ is being observed.

\section{Conclusions}

In this work, uncertainty analysis applied to a MYRRHA fuel assembly depletion case during whole fuel residence time was performed by propagating nuclear data covariances through the irradiation calculation. Firstly, it was observed a non-desirable effect in long-lived or stable fission products statistical variability (such the one of ${ }^{148} \mathrm{Nd}$ ) while depleting at constant power. The fact that the flux normalization is carried out at constant power, is bounding the effect of building up uncertainty in the fission product nuclide vector as a function of burnup. This effect was observed to be corrected if, instead, depletion steps were performed at constant flux. The addition of fission yield uncertainties (even at constant power) showed a large impact in the fission products inventory.

\section{References}

[1] H. Aït Abderrahim et al., Energy Conversion Management 63, p. 4-10 (2012)

[2] E. Malambu Mbala et al., SCK CEN/3958903 (2014)

[3] G. Van den Eynde et al., J. Nucl. Sci. Technol. 52 (7-8) p. 1053-1057 (2015)

[4] A. Stankovskiy and G. Van den Eynde, Sci. Technol. Nucl. Install, p. 545103 (2012)

[5] C.J. Werner (ed.), LA-UR-17-29981 (2017)

[6] S. Wook-Lee et al., Nucl. Eng. Technol. 46(4), p. 481488 (2014)

[7] J. Leppänen et al., Ann. Nucl. Energy 82, p. 142-150 (2014)

[8] A. Hernandez Solis et al., Proc. Int. Conf. PHYSOR2020, 37234070 (2020)

[9] M. B. Chadwick et al., Nucl. Data Sheets 112(2) p. 2887-2996 (2011)

[10] L. Fiorito et al., Ann. Nucl. Energy 101, p. 359-366 (2017)

[11] A. Santamarina et al., ISBN 978-92-64-99074-6, NEA No. 6807 (2009)

[12] K. Shibata et al., J. Nucl. Sci. Technol. 48(1), p. 1-30 (2011) 